\title{
Relativistic Quantum Theory for Charged Spinless Particles in External Vector Fields
}

\author{
Lars-Erik Lundberg \\ Nordita, Copenhagen. Denmark \\ Received February 5, 1973
}

\begin{abstract}
Guided by a diagonalized form of the classical field-energy we construct a time-dependent canonical pair of Schrödinger fields $\Phi_{t}(x)$ and $\Pi_{t}(x)$ which diagonalizes the field-Hamiltonian $H_{t}$. These Schrödinger fields in general belong to inequivalent representations of the canonical commutation relations for different $t$ 's.

The Heisenberg field is constructed by solving the Heisenberg equation of motion and its time-evolution turns out to be governed by a unitary operator, i.e. the Heisenberg fields at different times are unitarily equivalent.

Scattering theory (including eventual incoming and/or outgoing bound-states) is finally constructed.
\end{abstract}

\section{Introduction}

We shall in this paper develop a Hamiltonian formulation of relativistic quantum theory for charged spinless bosons in a local external vector potential $A_{\mu}(x, t)$. The formulation will be free from divergences.

External field problems in relativistic quantum theory has been studied frequently in the past and one naturally asks oneself if anything new can be added. In order to get a motivation for this work, let's briefly summarize what previously has been achieved.

Time-independent External Vector Potential: The solution of the external field problem in the time-independent case was essentially given by Heisenberg and Pauli [1] in their classical paper on quantum field theory. They proposed that one should quantize a classical field by expanding it in terms of the stationary solutions (eigenfunctions) and quantizing the normal coordinates (generalized Fourier-components). They actually only considered the Dirac case (spin $1 / 2$ ), but the KleinGordon case (spin 0) can be treated in complete analogy, see Schnyder and Weinberg [2].

The Hilbert-space $\mathscr{H}$, on which the fields are realized, is the Fockspace associated with the stationary modes, and the field-Hamiltonian $H$ and the charge-operator $Q$ define diagonal self-adjoint operators in $\mathscr{H}$ (when properly normal ordered) provided $A_{\mu}(x)$ is sufficiently regular. 
The Dirac case was considered in great detail by Moses [3] who attempted to implement the dynamics in the "free" Hilbert-space $\mathscr{H}_{0}$. This, however, was not possible when a magnetic field was present.

Bongaarts [4] made a rigorous derivation of Moses's results and developed scattering theory along the same lines as Lehmann, Symanzik, and Zimmermann [5] (although the language is somewhat different).

From this it is tempting to draw the following conclusion: A fully consistent field theory in the external field case is only obtained when the quantum field is realized on the Hilbert-space $\mathscr{H}$ where the full field-Hamiltonian $H$ and the charge-operator $Q$ take diagonal forms (provided $A_{\mu}(x)$ is sufficiently regular).

Time-dependent External Vector Potential: The time-dependent case is much more subtle than the time-independent case and the first successful treatment was given by Feynmann [6] with the so called space-time (or propagator) approach. This approach is based on an integrated version of the classical field-equation and allows any amplitude involving spin 0 or $1 / 2$ particles (and anti-particles) in an external vector potential, to be calculated to any order in the external field. The theory contains a divergence, the so called vacuum fluctuation, which however only give rise to an infinite phase-factor in any amplitude when all orders are taken into account.

A weak point in Feynmann's approach is that the proper statistics has to be introduced by hand. This difficulty, however, was solved by Dyson [7] by showing how to derive Feynmann's results from a perturbative formulation of quantum-field theory (including the vacuumfluctuation infinity).

Salam and Matthews [8] discussed exact solutions by studying a singular integral equation for the one-particle scattering amplitude (or pair production amplitude) which can be derived from the perturbationexpansion.

Schwinger [9] formulated the problem in terms of Green's functions and obtained the same results as Feynmann.

All these approaches have to our opinion the following drawback: The time-evolution of an initial state (as a vector in a Hilbert-space) cannot, as in non-relativistic quantum-mechanics, be considered unambiguously.

A covariant Hilbert-space formulation of the interaction of an electron-positron field (smeared over space-time) with an external vector potential (rapidly decreasing in space-time) has been given by Capri [10]. This theory, however, is manifestly a scattering theory in which the detailed dynamics (the time-evolution) cannot be considered. The interacting field has no physical interpretation, only the asymptotic fields can be interpreted. It is furthermore not clear how to generalize this 
approach to the case when $A_{\mu}(x, t)$ is not rapidly decreasing for $t \rightarrow \pm \infty$ (i.e. when one has possibilities for incoming and outgoing bound-states).

An attempt to study the time-evolution of a spinless charged field (smeared over space) interacting with an external vector potential was made by Schroer, Seiler and Swieca [11], when the field was realized on the incoming Hilbert-space. The result was negative, i.e. the field could not be realized in the interaction region when a magnetic field is present (this is consistent with Moses's observation [3] in the spin 1/2 case).

This result has lead Ekstein [12] to draw the following conclusion: "The physical interpretation of the intermediate or actual field is obscure. The concept of particles at finite times is not consistent with relativity and quantum theory."

Recently Labonté and Capri [13] made an important observation in the spin $1 / 2$ case. They noticed that the field-Hamiltonian can be diagonalized by introducing a time-dependent set of auxiliary fields which are solutions to a time-dependent set of stationary problems. They however argued that the auxiliary fields can be realized on the incoming Hilbert-space, which unfortunately turns out to be false in the general case (i.e. the incoming field and the auxiliary fields do in general belong to inequivalent representations of the canonical commutation relations).

We shall choose an approach to the general time-dependent case which is influenced by our conclusion in the summary of the timeindependent problem.

Guided by a diagonalized form of the classical field-energy we construct a time-dependent canonical pair of Schrödinger fields $\Phi_{t}(x)$ and $\Pi_{t}(x)$ which diagonalizes the field-Hamiltonian $H_{t}$. These Schrödinger fields do in general belong to inequivalent representations of the canonical commutation relations for different $t$ 's.

The Heisenberg field is constructed by solving the Heisenberg equation of motion and its time-evaluation turns out to be governed by a unitary operator i.e. the Heisenberg fields at different times are unitarily equivalent.

Time-evolution of states is then defined and the whole setup of quantum theory is constructed.

\section{The Classical Problem}

Before we can attack the quantized problem we shall need a detailed analysis of the Klein-Gordon (K-G) equation, when considered as a classical field-equation

$$
\left[-\left(i \partial_{\mu}-A_{\mu}\right)\left(i \partial^{\mu}-A^{\mu}\right)+m^{2}\right] u(x, t)=0,
$$

where the external vector-potential $A_{\mu}(x, t)$ is coupled minimally. 
The classical field-energy of a solution to (0.1) is given by

$$
\mathscr{E}_{A}\left(u, \partial_{t} u\right)=\int d x\left[|(\boldsymbol{\nabla}-i \boldsymbol{A}) u|^{2}+\left(m^{2}-A_{0}^{2}\right)|u|^{2}+\left|\partial_{t} u\right|^{2}\right],
$$

and is independent of $t$ when $A_{\mu}$ is.

It is essential for the consistency of our approach that $A_{\mu}$ is restricted such that ${ }^{1}$

$$
\varepsilon \mathscr{E}_{0}(u, v) \leqq \mathscr{E}_{A}(u, v) \leqq c \mathscr{E}_{0}(u, v),
$$

holds uniformly in $t$ for all $u, v \in C_{0}\left(R^{3}\right)$ (infinitely differentiable functions of compact support) and where $\varepsilon>0$ and $c \geqq 1$ are independant of $t, u$ and $v$ but may depend on $A_{\mu}$.

From any solution $u$ of $(0.1)$ we can construct a conserved current $j_{\mu}$ given by

$$
j_{\mu}=-i\left(\partial_{\mu} u\right)^{*} u+i u^{*} \partial_{\mu} u-2 A_{\mu} u^{*} u,
$$

i.e. $\partial^{\mu} j_{\mu}=0$ and the charge $Q$ defined by

$$
Q=\int d x j_{0},
$$

is independent of $t$.

\section{II.1. Spectral and Scattering Theory in the Stationary Case}

We shall in this section state the main results in [14], which were obtained in the case when $A_{\mu}=\delta_{\mu 0} A_{0}$, but can easily be generalized to the present case (see Appendix A).

Equation (0.1) can in the stationary be written

$$
i \partial_{t} \Psi=B \Psi
$$

where $\Psi=\left(\begin{array}{c}u \\ i \partial_{t} u\end{array}\right)$ and

$$
B=\left(\begin{array}{cc}
0 & 1 \\
L_{A} & 2 A_{0}
\end{array}\right), \quad L_{A}=(-i \boldsymbol{\nabla}-\boldsymbol{A})^{2}-A_{0}^{2}+m^{2} .
$$

Let $f=\left(\begin{array}{l}f_{1} \\ f_{2}\end{array}\right)$ and $g=\left(\begin{array}{l}g_{1} \\ g_{2}\end{array}\right) \in \mathscr{D}=C_{0}^{\infty}\left(R^{3}\right) \times C_{0}^{\infty}\left(R^{3}\right)$ and put

$$
(f, g)_{\mathscr{E}}=\int d x\left[(i \boldsymbol{\nabla}-\boldsymbol{A}) f_{1}^{*}(-i \boldsymbol{\nabla}-\boldsymbol{A}) g_{1}+\left(m^{2}-A_{0}^{2}\right) f_{1}^{*} g_{1}+f_{2}^{*} g_{2}\right] \text {, }
$$

i.e. $\mathscr{E}_{A}\left(u, \partial_{t} u\right)=(\Psi, \Psi)_{\mathscr{E}}$.

\footnotetext{
1 This condition also prevents the so called Klein-Paradox to occur.
} 
The positivity of the classical field-energy (0.2) allows us to construct a Hilbert space $\mathscr{H}_{\mathscr{E}}$ by completeing $\mathscr{D}$ in the norm $\|f\|_{\mathscr{E}}=\sqrt{(f, f)_{\mathscr{E}}}$ and with (1.3) as scalar product. It follows from (0.3) that $\mathscr{H}_{\mathscr{E}}$ is given by

$$
\mathscr{H}_{\mathscr{E}}=\mathscr{H}^{1} \times L^{2}\left(R^{3}\right),
$$

where $\mathscr{H}^{p}$ stands for the Sobolev space containing those functions for which the function and all derivatives up to (including) $p$-th order are square-integrable.

Provided the initial-value problem given by (1.1) is well posed, it follows that the time-evolution operator $U(t)(\Psi(t)=U(t) \Psi(0))$ defines a unitary one-parameter group in $\mathscr{H}_{\mathscr{E}}$ (follows from conservation of energy).

We shall from now on assume that the conditions on $A_{\mu}$ given in Appendix A are fulfilled.

The operator $B$ defines a self-adjoint operator on $D(B)=\mathscr{H}^{2} \times \mathscr{H}^{1}$ with the following spectrum

$$
\sigma_{\mathrm{e}}(B)=\sigma_{\text {a.c. }}(B)=(-\infty,-m) \cup[m, \infty),
$$

where e(a.c.) stands for essential (absolutely continuous).

The differential Eq. (1.1) can now be integrated

$$
\Psi(t)=e^{-i B t} \Psi(0)=\int_{-\infty}^{\infty} e^{-i \lambda t} d E(\lambda) \Psi(0),
$$

where we have employed the spectral representation for $B, B=\int_{-\infty}^{\infty} \lambda d E(\lambda)$ and we have assumed that $\Psi(0) \in D(B)$.

Let us for future purposes define the following projection operators

$$
P^{+}=1-E(0), \quad P^{-}=E(0), \quad P_{c}^{+}=1-E(m), \quad P_{c}^{-}=E(-m) .
$$

The spectral family $E(\lambda)$ can be expressed in terms of eigenfunctions $\Phi_{n}^{ \pm}(\cdot)$ and $\Phi^{ \pm}(\cdot, k)$ of $B$ with eigenvalues $\omega_{n}^{ \pm} \gtrless 0, n=1, \ldots, n^{ \pm}$and $\pm \omega_{k}= \pm \sqrt{k^{2}+m^{2}}$ respectively. These eigenfunctions are normalized as follows

$$
\left(\Phi_{n}^{ \pm}, \Phi_{m}^{ \pm}\right)_{\mathscr{E}}=\delta_{n m},\left(\Phi^{ \pm}(\cdot, k), \Phi^{ \pm}(\cdot, k)\right)_{\mathscr{E}}=\delta\left(k-k^{\prime}\right),
$$

and have the following explicit form

$$
\Phi_{n}^{ \pm}(x)=\left(\begin{array}{c}
u_{n}^{ \pm}(x) \\
\omega_{n}^{ \pm} u_{n}^{ \pm}(x)
\end{array}\right), \quad \Phi^{ \pm}(x, k)=c_{k}\left(\begin{array}{c}
u^{ \pm}(x, k) \\
\pm \omega_{k} u^{ \pm}(x, k)
\end{array}\right),
$$


where $u_{n}^{ \pm}(x)$ and $u^{ \pm}(x, k)$ are solutions of the stationary form of $(0.1)$ (and $c_{k}$ is given by $c_{k}=\frac{1}{(2 \pi)^{3 / 2}} \frac{1}{\sqrt{2 \omega_{k}}}$ )

$$
\left(-\omega^{2}+2 \omega A_{0}+L_{A}\right) u(x)=0 \text {, }
$$

with $\omega=\omega_{n}^{ \pm}$and $\omega= \pm \omega_{k}$ respectively, i.e. $u_{n}^{ \pm}(x)$ are square integrable solutions and $u^{ \pm}(x, k)$ are solutions of the following integrated version of (1.8)

$$
u^{ \pm}(x, k)=e^{i k x}-\frac{1}{4 \pi} \int d y \frac{e^{ \pm i|k||x-y|}}{|x-y|} V^{ \pm}(y, k) u^{ \pm}(y, k),
$$

where

$$
V^{ \pm}(y, k)= \pm 2 \omega_{k} A_{0}(y)+2 i \boldsymbol{A}(y) \cdot \boldsymbol{\nabla}+i(\boldsymbol{\nabla} \cdot \boldsymbol{A}(y))-A_{\mu}(y)^{2} .
$$

Let us furthermore define the following "Fourier-transforms" of $f \in \mathscr{D}$

$$
\hat{f}_{n}^{ \pm}=\left(\Phi_{n}^{ \pm}, f\right)_{\delta}, \quad \hat{f}^{ \pm}(k)=F^{ \pm} f(k)=\left(\Phi^{ \pm}(\cdot, k), f\right)_{\mathscr{E}} .
$$

The spectral projection $E(\lambda)$ (associated with $B$ ) can be represented as follows $(f, g \in \mathscr{D})$

$$
\begin{aligned}
(g, E(\lambda) f)_{\delta} & =\int_{-\omega_{k}<\lambda} d k \hat{g}^{-*}(k) \hat{f}^{-}(k) \quad(\lambda<-m) \\
& =\int d k \hat{g}^{-*}(k) \hat{f}^{-}(k)+\sum_{\omega_{n}^{ \pm}<\lambda} \hat{g}_{n}^{ \pm *} \hat{f}_{n}^{ \pm} \quad(-m<\lambda<m) \\
& =\int d k \hat{g}^{-*}(k) \hat{f}^{-}(k)+\sum_{n} \hat{g}_{n}^{ \pm *} \hat{f}_{n}^{ \pm}+\int_{\omega_{k}<\lambda} d k \hat{g}^{+*}(k) \hat{f}^{+}(k)
\end{aligned}
$$

which exhibits the completeness of $\Phi_{n}^{ \pm}(\cdot)$ and $\Phi^{ \pm}(\cdot, k)$.

In the quantized theory we shall need the following sesquilinear form

$$
(f, g)_{Q}=\left(f, B^{-1} g\right)_{\mathscr{E}}=\int d x\left(f_{1}^{*} g_{2}+f_{2}^{*} g_{1}-2 A_{0} f_{1}^{*} g_{1}\right),
$$

and for future convenience we introduce $\bar{f}=\left(\begin{array}{c}f_{1} \\ f_{2}-A_{0} f_{1}\end{array}\right)$ which means that (1.11) can be written

$$
(f, g)_{Q}=\left(\bar{f}_{1}, \bar{g}_{2}\right)_{2}+\left(\bar{f}_{2}, \bar{g}_{1}\right)_{2} \equiv(\bar{f}, \bar{g})_{\sigma},
$$

where 2 stands for $L^{2}\left(R^{3}\right)$. 
It follows from (1.11) that $B^{-1}$ is bounded in $\mathscr{H}_{\mathscr{E}}$. This means that we can complete $\mathscr{H}_{\delta}$ in the norm \|\|$_{I}$ defined by

$$
\|f\|_{I}^{2}=(f, f)_{I}=\frac{1}{2}\left(f,|B|^{-1} f\right)_{\mathscr{E}},
$$

and get a Hilbert-space $\mathscr{H}_{I} \supset \mathscr{H}_{\mathscr{E}}$. We notice that $(f, g)_{Q}$ is bounded for all $f, g \in \mathscr{H}_{I}$ and that $(\Psi, \Psi)_{Q}=Q$ with $Q$ given by $(0.5)$.

Let $B_{0}, \mathscr{H}_{\mathscr{E}_{0}}, \mathscr{H}_{I_{0}}, P_{0}^{ \pm}$and $F_{0}^{ \pm}$denote $B, \mathscr{H}_{\mathscr{E}}, \mathscr{H}_{I}, P_{c}^{ \pm}$and $F^{ \pm}$respectively when $A_{\mu}=0$.

One can prove (under our conditions on $A_{\mu}$ ) that the wave-operators $W_{ \pm}=W_{ \pm}\left(B, B_{0}\right)$ defined by

$$
W_{ \pm}=\underset{t \rightarrow \pm \infty}{s-\lim _{t \rightarrow \infty}} e^{i B t} e^{-i B_{0} t},
$$

both exist and map $P_{0}^{ \pm} \mathscr{H}_{\mathscr{E}_{0}}$ isometrically onto $P_{\mathrm{c}}^{ \pm} \mathscr{H}_{\mathscr{E}}$. They furthermore intertwine $B_{0}$ and $B_{c}=\left(P_{c}^{+}+P_{c}^{-}\right) B$, i.e.

$$
W_{ \pm} B_{0} \subset B_{c} W_{ \pm} \text {. }
$$

The following representation of $W_{ \pm}$holds

$$
P_{c}^{ \pm} W_{-}=F^{ \pm *} F_{0}^{ \pm}, \quad W_{+} f=\left(W_{-} f^{*}\right)^{*},
$$

(here * stands both for adjoint and complex conjugate) and the scattering operator $S$ is defined by

$$
S=W_{+}^{*} W_{-} P_{0}^{+}+W_{-}^{*} W_{+} P_{0}^{-} \equiv S^{+}+S^{-},
$$

and is easily seen to be unitary in $\mathscr{H}_{\mathscr{E}_{0}}$ and commutes with $B_{0}$. This implies that $S$ also defines a unitary operator in $\mathscr{H}_{I_{0}}$.

By inserting (1.16) into (1.17) we get the ordinary stationary definition of the $S$-matrix (see [14]).

\section{II.2. Solution of the Initial-value Problem in the Time-dependent Case}

The K-G equation (0.1) can in the general case be written

$$
i \partial_{t} \Psi=\tilde{B}_{t} \Psi,
$$

with $\Psi=\left(\begin{array}{c}u \\ i \partial_{t} u\end{array}\right)$ and

$$
\tilde{B}_{t}=\left(\begin{array}{cc}
0 & 1 \\
\tilde{L}_{t} & 2 A_{0}
\end{array}\right) \tilde{L}_{t}=(-i \boldsymbol{\nabla}-\boldsymbol{A})^{2}-A_{0}^{2}+i \partial_{t} A_{0}+m^{2} .
$$


Let us define

$$
B_{t}=\left(\begin{array}{cc}
0 & 1 \\
L_{t} & 2 A_{0}
\end{array}\right) L_{t}=(-i \boldsymbol{\nabla}-\boldsymbol{A})^{2}-A_{0}^{2}+m^{2},
$$

and denote by $\mathscr{H}_{\mathscr{E}_{t}}$ the Hilbert-space (of the kind discussed in the previous section) where $B_{t}$ is self-adjoint. It follows from $(0.3)$ that $\mathscr{H}_{\mathscr{E}_{t}}$ and $\mathscr{H}_{\mathscr{E}_{t^{\prime}}}$ are equivalent as Banach-spaces i.e. any vector in $\mathscr{H}_{\mathscr{E}_{t}}$ also belongs to $\mathscr{H}_{\mathscr{E}^{\prime}}$ for all $t$ and $t^{\prime}$.

We shall assume that $A_{\mu}$ fulfills the conditions given in Appendix A uniformly in $t$ and furthermore is such that $V_{t t^{\prime}}$ defined by

$$
\tilde{B}_{t}=B_{t^{\prime}}+V_{t t^{\prime}}
$$

is bounded in $\mathscr{H}_{I_{t}}$. This implies that we have the following representation for the time-evolution operator $U\left(t, t^{\prime}\right)$ associated with Eq. (2.1)

$$
U\left(t, t^{\prime}\right)=e^{-i B_{t^{\prime}}\left(t-t^{\prime}\right)} T\left(e^{-i \int_{t^{\prime}}^{t} d t \tilde{V}_{t t^{\prime}}}\right),
$$

(where $T$ stands for time-ordering) with

$$
\tilde{V}_{t t^{\prime}}=e^{i B_{t^{\prime}}\left(t-t^{\prime}\right)} V_{t t^{\prime}} e^{-i B_{t^{\prime}}\left(t-t^{\prime}\right)} .
$$

The time-evolution operator $U\left(t, t^{\prime}\right)$ is not in general unitary in any Hilbert-space but have instead the following property

$$
\left(U\left(t, t^{\prime}\right) f, U\left(t, t^{\prime}\right) g\right)_{Q_{t}}=(f, g)_{Q_{t^{\prime}}},
$$

which we shall call charge-isometry, i.e. it leaves the charge $Q$ invariant $\left((,)_{Q_{t}}\right.$ is given by $(1.11)$ with $\left.B=B_{t}\right)$.

\section{II.3. Diagonal Representation of the Field-energy}

The field-energy (0.2) has the same form in the time-dependent case as in the stationary case i.e.

$$
\mathscr{E}_{A}=(\Psi(t), \Psi(t))_{\mathscr{E}_{t}},
$$

and takes diagonal form if $\Psi(t)$ is expanded in terms of eigenfunctions of $B_{t}$

with

$$
\mathscr{E}_{A}=\sum_{ \pm}\left[\sum_{n=1}^{n^{ \pm}(t)} \hat{\Psi}_{t, n}^{ \pm *} \hat{\Psi}_{t, n}^{ \pm}+\int d k \hat{\Psi}_{t}^{ \pm *}(k) \hat{\Psi}_{t}^{ \pm}(k)\right],
$$

$$
\hat{\Psi}_{t, n}^{ \pm}=\left(\Phi_{t, n}^{ \pm}, \Psi(t)\right)_{\mathscr{E}_{t}}, \quad \hat{\Psi}_{t}^{ \pm}(k)=\left(\Phi_{t}^{ \pm}(\cdot, k), \Psi(t)\right)_{\mathscr{E}_{t}}
$$




\section{The Quantized Problem}

A Hamiltonian formulation will in this section be employed in order to construct a quantum-field theory associated with the classical fieldequation (0.1).

The diagonalized classical field-energy (II.3.2) will be the starting point for the whole construction.

\section{III.1. Construction of the Quantum-field, the Current,} the Charge-operator and the Number-operator

in the Diagonal Hamiltonian Schrödinger Picture (D.H.S.P.)

The diagonal representation (II.3.2) of the classical field-energy can be written

$\mathscr{E}_{t}=\sum_{ \pm}\left[\sum_{n=1}^{n^{ \pm}(t)} \frac{1}{2\left|\omega_{n}^{ \pm}\right|}\left|\omega_{n}^{ \pm}\right| a_{n}^{ \pm *}(t) a_{n}^{ \pm}(t)+\int \frac{d k}{2 \omega_{k}} \omega_{k} a^{ \pm *}(k, t) a^{ \pm}(k, t)\right]$,

where

$$
\begin{gathered}
a_{n}^{ \pm}(t)=\sqrt{2} \hat{\Psi}_{t, n}^{ \pm}=\sqrt{2}\left(\Phi_{t, n}^{ \pm}, \Psi(\cdot, t)\right)_{\mathscr{E}_{t}}, \\
a^{ \pm}(k, t)=\sqrt{2} \hat{\Psi}_{t}^{ \pm}(k)=\sqrt{2}\left(\Phi_{t}^{ \pm}(\cdot, k), \Psi(\cdot, t)\right)_{\mathscr{E}_{t}} .
\end{gathered}
$$

The time-dependence of $a_{n}^{ \pm}(t)$ and $a^{ \pm}(k, t)$ is in the stationary case given by a phase-factor $e^{-i \omega_{n}^{ \pm} t}$ and $e^{\mp i \omega_{k} t}$ respectively i.e. $\mathscr{E}_{t}$ is timeindependent $\left(\left\{\omega_{n}^{ \pm}\right\}_{n=1}^{n^{ \pm}(t)}\right.$ are the eigenvalues of $\left.B_{t}\right)$.

Let us now by an ordinary Fock-Cook construction introduce a Hilbert-space $\mathscr{H}_{t}$ on which the following creation and annihilation"operators" are defined (for a proper definition see below)

$\left[a_{n}^{ \pm}(t), a_{m}^{ \pm *}(t)\right]=2\left|\omega_{n}^{ \pm}\right| \delta_{n m}, \quad\left[a^{ \pm}(k, t), a^{ \pm *}\left(k^{\prime}, t\right)\right]=2 \omega_{k} \delta\left(k-k^{\prime}\right)$,

with all other commutators vanishing and furthermore having the property that there exists a vacuum state $|0\rangle_{t} \in \mathscr{H}_{t}$, i.e.

$$
a_{n}^{ \pm}(t)|0\rangle_{t}=a^{ \pm}(k)|0\rangle_{t}=0 .
$$

Let us for $f \in \mathscr{H}_{I_{t}}$ define $a_{t}^{ \pm}(f)$ by

$$
a_{t}^{ \pm}(f)=\sum_{n=1}^{n \pm(t)} \frac{1}{2\left|\omega_{n}^{ \pm}\right|} \hat{f}_{n}^{ \pm *} a_{n}^{ \pm}(t)+\int \frac{d k}{2 \omega_{k}} \hat{f}^{ \pm *}(k) a^{ \pm}(k, t),
$$

which is easily seen to define a closed operator in $\mathscr{H}_{t}$. The proper operator form of (1.3) and (1.4) then becomes

$$
\left[a_{t}^{ \pm}(f), a_{t}^{ \pm}(g)^{*}\right]=\left(f, P_{t}^{ \pm} g\right)_{I_{t}}, \quad a_{t}^{ \pm}(f)|0\rangle_{t}=0,
$$


with all other commutators vanishing $\left(P_{t}^{ \pm}\right.$are projection-operators associated with $B_{t}$ [see (II.1.5)]).

The structure of the Hilbert-space $\mathscr{H}_{t}=\mathscr{H}$ is the following

$$
\mathscr{H}=\mathscr{H}_{0} \oplus \sum_{ \pm} \sum_{n=1}^{\infty} \oplus\left(\mathscr{H}_{n, c}^{ \pm} \oplus \mathscr{H}_{n, d}^{ \pm}\right)
$$

where $\mathscr{H}_{0}$ is spanned by the vacuum state $|0\rangle_{t}$ and $\mathscr{H}_{n, c}^{ \pm}$by symmetric functions (defined on $R^{3 n}$ ) with the scalar product given by

$$
(f, g)_{n, c}=\frac{1}{n !} \int \frac{d k_{1}}{2 \omega_{k_{1}}} \ldots \frac{d k_{n}}{2 \omega_{k_{n}}} f\left(k_{1}, \ldots, k_{n}\right)^{*} g\left(k_{1}, \ldots, k_{n}\right),
$$

and $\mathscr{H}_{n, d}^{ \pm}$is spanned by symmetric $n^{ \pm}(t)$ dimensional tensors of rank $n$ with the scalar product

$$
(f, g)_{d, c}=\frac{1}{n !} \sum_{\alpha_{1}, \ldots, \alpha_{n}}^{n \pm(t)} \frac{1}{2 \omega_{\alpha_{1}}} \ldots \frac{1}{2 \omega_{\alpha_{n}}} f_{\alpha_{1} \ldots \alpha_{n}}^{*} g_{\alpha_{1} \ldots \alpha_{n}} .
$$

The classical field-energy (1.1) is easily seen to define a non-negative diagonal self-adjoint operator $H_{t}$ in $\mathscr{H}_{t}$, when the functions $a_{n}^{ \pm}(t)$ and $a^{ \pm}(k, t)$ are replaced with the corresponding Fock-operators (denoted with the same symbols)

$$
H_{t}=\sum_{ \pm}\left[\sum_{n=1}^{n \pm(t)} \frac{1}{2\left|\omega_{n}^{ \pm}\right|}\left|\omega_{n}^{ \pm}\right| N_{n}^{ \pm}(t)+\int \frac{d k}{2 \omega_{k}} \omega_{k} N^{ \pm}(k, t)\right],
$$

where $N_{n}^{ \pm}(t)=a_{n}^{ \pm}(t)^{*} a_{n}^{ \pm}(t)$ and $N^{ \pm}(k, t)=a^{ \pm}(k, t)^{*} a^{ \pm}(k, t)$.

It is essential that the creation operator $a^{*}$ is placed to the left of the annihilation-operator a upon quantization (this is usually called normal ordering).

The Hamiltonian $H_{t}$ has 0 as a non-degenerate eigenvalue with the vacuum-state $|0\rangle_{t}$ as eigenvector.

The time-dependence of the $a(t)$ 's in the classical case is upon quantization absorbed into the Hilbert-space $\mathscr{H}_{t}$ and we shall therefore call $a_{t}^{ \pm}(f)$ operators in the diagonal field-Hamiltonian Schrödinger picture (D.H.S.P.).

Guided by the relation (1.2) between the classical field $\Psi(x, t)$ and the $a(t)$ 's we will now define the quantum-field $\Psi(x, t)=\left(\begin{array}{c}\Phi(x, t) \\ i \partial_{t} \Phi(x, t)\end{array}\right)$ in the D.H.S.P. by

$$
\begin{aligned}
(f, \Psi(\cdot, t))_{Q_{t}}=(\bar{f}, \bar{\Psi}(\cdot, t))_{\sigma} & =\sqrt{2}\left(a_{t}^{+}(f)+a_{t}^{-}\left(f^{*}\right)^{*}\right) \\
& =\Psi_{t}(f)=\bar{\Psi}_{t}(\bar{f}),
\end{aligned}
$$

where we have used (II.1.12). 
The commutation relations (1.6) gives

$$
\begin{aligned}
{\left[\Psi_{t}(f), \Psi_{t}(g)^{*}\right] } & =\left[\bar{\Psi}_{t}(\bar{f}), \bar{\Psi}_{t}(\bar{g})^{*}\right] \\
& =(f, g)_{Q_{t}}=(\bar{f}, \bar{g})_{\sigma},
\end{aligned}
$$

and $\left[\Psi_{t}(f), \Psi_{t}(g)\right]=0$. Thus the commutator $\left[\bar{\Psi}_{t}(f), \bar{\Psi}_{t}(g)^{*}\right]$ is independent of $t$. This could suggest that $\bar{\Psi}_{t}(f)$ and $\bar{\Psi}_{t^{\prime}}(f)$ are unitarily equivalent, which however turns out to be false in the general case, i.e. $\bar{\Psi}_{t}(f)$ and $\bar{\Psi}_{t^{\prime}}(f)$ do in general belong to inequivalent representations of the commutation relations.

The commutation relations (1.12) can be put into a more familiar form

$$
\begin{gathered}
{\left[\Phi_{t}(x), \Pi_{t}(y)\right]=i \delta(x-y),} \\
{\left[\Phi_{t}(x), \Phi_{t}(y)\right]=\left[\Pi_{t}(x), \Pi_{t}(y)\right]=0,}
\end{gathered}
$$

where $\Phi_{t}(x)=\Phi(x, t)$ and $\Pi_{t}(x)$ denotes the field canonically conjugate to $\Phi_{t}(x)$, i.e.

$$
\Pi_{t}(x)=\partial_{t} \Phi^{*}(x, t)-i A_{0}(x, t) \Phi^{*}(x, t) .
$$

Let us close this section by defining the current, the charge operator and the number operator in the D.H.S.P.

The classical current (II.0.4) does upon quantization define a sesquilinear form (which means that matrix-elements of the current has proper meaning) which is densely defined provided the current is normal ordered

$$
\begin{aligned}
j_{0}(x)_{t} & =:\left(-i \Pi_{t}(x) \Phi_{t}(x)+i \Phi_{t}(x) \Pi_{t}(x)\right): \\
\boldsymbol{j}_{t}(x) & =:\left(-i\left(\boldsymbol{\nabla} \Phi_{t}(x)\right) \Phi_{t}(x)+i \Phi_{t}(x) \boldsymbol{\nabla} \Phi_{t}(x)\right),
\end{aligned}
$$

where the dots : : stands for normal ordering.

The charge (II.0.5) in the classical theory does upon quantization in the D.H.S.P. take the following form

$$
Q_{t}=\sum_{ \pm} \pm\left[\sum_{n=1}^{n \pm(t)} \frac{1}{2\left|\omega_{n}^{ \pm}\right|} N_{n}^{ \pm}(t)+\int \frac{d k}{d \omega_{k}} N^{ \pm}(k, t)\right]
$$

and the number-operator is finally defined by

$$
N_{t}=\sum_{ \pm}\left[\sum_{n=1}^{n \pm(t)} \frac{1}{2\left|\omega_{n}^{ \pm}\right|} N_{n}^{ \pm}(t)+\int \frac{d k}{2 \omega_{k}} N^{ \pm}(k, t)\right]
$$




\section{III.2. Construction of the Quantum-field}

in the Diagonal Field-Hamiltonian Heisenberg Picture (D.H.H.P.) and Existence of the Time-evolution Operator

We shall in this section construct the Heisenberg field $\Psi\left(f, t, t_{0}\right)$ as a solution of the Heisenberg equation of motion

$$
i \frac{d}{d t} \Psi\left(f, t, t_{0}\right)=\left[\Psi\left(f, t, t_{0}\right), H_{t}\right]
$$

with the initial condition $\Psi\left(f, t_{0}, t_{0}\right)=\Psi_{t_{0}}(f)$ and fulfilling the following equal-time commutation relations

$$
\left[\Psi\left(f, t, t_{0}\right), \Psi\left(g, t, t_{0}\right)^{*}\right]=(f, g)_{Q_{t_{0}}}, \quad\left[\Psi\left(f, t, t_{0}\right), \Psi\left(g, t, t_{0}\right)\right]=0 .
$$

The occurrence of $H_{t}$ in (2.1) forces us to realize $\Psi\left(f, t, t_{0}\right)$ on $\mathscr{H}_{t}$ and the canonical structure (2.2) suggests that $\Psi\left(f, t, t_{0}\right)$ contains $\Phi_{t}$ and $\Pi_{t}$ linearily.

The total derivative $\frac{d}{d t}$ is assumed not to act on $\Phi_{t}$ and $\Pi_{t}$.

The solution of (2.1) and (2.2) is given by

$$
\Psi\left(f, t, t_{0}\right)=\Psi_{t}\left(\bar{U}\left(t, t_{0}\right) f\right),
$$

where $\bar{U}\left(t, t_{0}\right)$ is a solution of

$$
i \partial_{t} \bar{U}\left(t, t_{0}\right)=-\bar{B}_{t} \bar{U}\left(t, t_{0}\right), \bar{U}\left(t_{0}, t_{0}\right)=1,
$$

with $\bar{B}_{t}$ given by

$$
\bar{B}_{t}=\left(\begin{array}{cc}
0 & 1 \\
\bar{L}_{t} & 2 A_{0}
\end{array}\right), \quad \bar{L}_{t}=(-i \boldsymbol{\nabla}-\boldsymbol{A})^{2}-A_{0}^{2}-i \partial_{t} A_{0}+m^{2} .
$$

One can easily verify that $\bar{U}\left(t, t_{0}\right)$ is charge-isometric.

The proof of (2.3) is obtained by inserting it into (2.1) and using (1.11), i.e. (2.1) holds provided

$$
i \frac{d}{d t} \overline{\bar{U}\left(t, t_{0}\right) f}=-\overline{B_{t} \bar{U}\left(t, t_{0}\right) f} .
$$

This equation can be written

$$
i \frac{\partial}{\partial t} \bar{U}\left(t, t_{0}\right) f=-\bar{B}_{t} \bar{U}\left(t, t_{0}\right) f,
$$

and thus coincides with (2.4). The charge-isometry of $\bar{U}\left(t, t_{0}\right)$ and (1.12) proves (2.2). 
The Heisenberg fields $\Psi\left(f, t, t_{0}\right)$ are unitarily equivalent for different $t\left(t_{0}\right.$ fixed) i.e. there exists a unitary operator $\mathscr{U}\left(t, t_{0}\right)$ from $\mathscr{H}_{t_{0}}$ onto $\mathscr{H}_{t}$ such that

$$
\Psi\left(f, t, t_{0}\right)=\mathscr{U}\left(t, t_{0}\right) \Psi\left(f, t_{0}, t_{0}\right) \mathscr{U}\left(t, t_{0}\right)^{-1} .
$$

Due to the fact that $\mathscr{U}\left(t, t_{0}\right)$ leaves the commutation relations (2.2) invariant, it follows that it is sufficient for the unitary of $\mathscr{U}\left(t, t_{0}\right)$ that there exists a vector $|0\rangle_{t t_{0}}$ in $\mathscr{H}_{t}$ with the property

$$
\Psi\left(f_{0}^{+}, t, t_{0}\right)|0\rangle_{t t_{0}}=\Psi\left(f_{0}^{-}, t, t_{0}\right)^{*}|0\rangle_{t t_{0}}=0,
$$

i.e. $|0\rangle_{t t_{0}}=\mathscr{U}\left(t, t_{0}\right)|0\rangle_{t_{0}}\left(f_{0}^{ \pm}=P_{t_{0}}^{ \pm} f\right.$ and $\left.f \in \mathscr{H}_{I_{t_{0}}}\right)$.

Equations (2.7), (2.3), (1.11) and (1.5) gives

$$
\left(a_{t}^{+}(g)+a_{t}^{-}\left((K g)^{*}\right)^{*}\right)|0\rangle_{t t_{0}}=0,
$$

with

$$
K=\left(1-K_{1}\right)^{-1} K_{2},
$$

where

$$
K_{1}=P_{t}^{-} \bar{U} P_{t_{0}}^{+} \bar{U}^{-1} P_{t}^{-}, \quad K_{2}=P_{t}^{-} \bar{U} P_{t_{0}}^{+} \bar{U}^{-1} P_{t}^{+},
$$

provided $\bar{U} \mathscr{H}_{I_{t_{0}}}^{+}$is dense in $\mathscr{H}_{I_{t}}^{+}$. A necessary and sufficient condition for (2.8) is that $K$ is Hilbert-Schmidt in $\mathscr{H}_{I_{t}}$ with a norm less than one, i.e.

$$
K=\sum_{i=1}^{\infty} \lambda_{i} f_{i}^{-} \otimes f_{i}^{+}
$$

where $\sum_{i=1}^{\infty} \lambda_{i}^{2}<\infty, 0<\lambda_{i}<1$ and $\left\{f_{i}^{ \pm}\right\}_{i=1}^{\infty}$ is an orthonormal set in $\mathscr{H}_{I_{t}^{ \pm}}^{ \pm}$.

The representation (2.11) of $K$ then allows an explicit construction of $|0\rangle_{t t_{0}}$ i.e.

with

$$
|0\rangle_{t t_{0}}=c \prod_{i=1}^{\infty} e^{-\lambda_{2} a_{l}^{+*} a_{i}^{-*}}|0\rangle_{t}
$$

$$
c^{2}=\prod_{i=1}^{\infty}\left(1-\lambda_{i}^{2}\right), a_{i}^{ \pm}=a_{t}^{ \pm}\left(f_{i}^{ \pm}\right) .
$$

We have thus reduced the problem of unitarity of $\mathscr{U}\left(t, t_{0}\right)$ to the following properties of $\bar{U}=\bar{U}\left(t, t_{0}\right)$ and $K=K\left(t, t_{0}\right)$;

i) $\bar{U} \mathscr{H}_{I_{t_{0}}}^{+}$is dense in $\mathscr{H}_{I_{t}}^{+}$,

ii) $\|K\|_{I_{t}}<1$,

iii) $K$ is Hilbert-Schmidt in $\mathscr{H}_{I_{t}}$.

These properties are verified, under our conditions on the external potential $A_{\mu}$, in Appendix B. 


\section{III.3. Definition of Physical States and Their Time-evolution in the D.H.S.P.}

The definition of physical particle states at $t=t_{0}$ is straight-forward due to the fact that the Hilbert-space $\mathscr{H}_{t_{0}}$ is buildt up by a Fock-Cook construction. The structure of the Hilbert-space is given in (1.7) and the only novel part, compared with the free case, is that we might have bound states.

Let us assume that the system is in a state $\left|\xi\left(t_{0}\right)\right\rangle \in \mathscr{H}_{t_{0}}$ at $t=t_{0}$. We shall define the corresponding time-evolved state $|\xi(t)\rangle \in \mathscr{H}_{t}$ in the D.H.S.P. by

$$
|\xi(t)\rangle=\mathscr{U}\left(t, t_{0}\right)\left|\xi\left(t_{0}\right)\right\rangle .
$$

Note that we have chosen the inverse of the usual time-evolution operator in the Schrödinger picture, as our time-evolution operator for a state-vector in the D.H.S.P. The reason for this choice is that $\mathscr{U}\left(t, t_{0}\right)$ maps $\mathscr{H}_{t_{0}}$ onto $\mathscr{H}_{t}$ and we naturally want the initial state to be defined in $\mathscr{H}_{t_{0}}$.

In Section III.1 we defined the operators $H_{t}, Q_{t}$ and $N_{t}$ in the D.H.S.P. as certain self-adjoint operators on $\mathscr{H}_{t}$. Let us consider the expectationvalues of these operators in time-evolving states of the type (3.1). For simplicity we consider the state $|0\rangle_{t t_{0}}$ obtained when $\left|\xi\left(t_{0}\right)\right\rangle$ in (3.1) is chosen equal to $|0\rangle_{t_{0}}$.

The expectation value of the Hamiltonian $H_{t}$ in this state

$$
\left\langle H_{t}\right\rangle={ }_{t t_{0}}\left\langle 0\left|H_{t}\right| 0\right\rangle_{t t_{0}},
$$

can be estimated by using the representation (2.11) for $|0\rangle_{t t_{0}}$ and the estimate for the kernel of $K\left(t, t_{0}\right)$ given in Appendix B. It is in general not finite in the interaction-region, i.e. the local external vector potential $A_{\mu}(x, t)$ might pump in infinite amount of energy into a physical system.

The expectation value of the number-operator

$$
\left\langle N_{t}\right\rangle={ }_{t t_{0}}\left\langle 0\left|N_{t}\right| 0\right\rangle_{t t_{0}},
$$

however is always finite, i.e. even when an infinite amount of energy is pumped into the system, it is a finite average number of particles that get that infinite average energy ${ }^{2}$.

The expectation-value of the charge is always constant i.e.

$$
\mathscr{U}\left(t, t_{0}\right) Q_{t_{0}} \subset Q_{t} \mathscr{U}\left(t, t_{0}\right),
$$

(for the proof of these statements see below).

2 This is directly related to the divergences in the case of interacting fields. 
In the case when $A_{\mu}(x, t)$ is time-dependent, it is convenient to expand the Schrödinger-fields in the orthonormal set $\left\{f_{i}^{ \pm}\right\}_{i=1}^{\infty}$ associated with $K\left(t, t_{0}\right)($ see $(2.11))$, i.e. $a_{t}^{ \pm}(f)$ given by $(1.5)$ has the following representation

$$
a_{t}^{ \pm}(f)=\sum_{i=1}^{\infty} f_{i}^{ \pm *} a_{i}^{ \pm}+a_{t}^{ \pm}(f)^{\perp},
$$

where $a_{t}^{ \pm}(f)^{\perp}=0$ if $\left\{f_{i}^{ \pm}\right\}_{i=1}^{\infty}$ happens to be complete in $P_{t}^{ \pm} \mathscr{H}_{I_{t}}$.

The charge-operator $Q_{t}$ and the number-operator $N_{t}$ can similarly be written

$$
Q_{t}=\sum_{ \pm} \pm \sum_{i=1}^{\infty} a_{i}^{ \pm *} a_{i}^{ \pm}+Q_{t}^{\perp}, \quad N_{t}=\sum_{ \pm} \sum_{i=1}^{\infty} a_{i}^{ \pm *} a_{i}^{ \pm}+N_{t}^{\perp} .
$$

Equations (2.11) and (3.6) give

$$
Q_{t}|0\rangle_{t_{0}}=Q_{t_{0}}|0\rangle_{t_{0}}=0,
$$

which proves that (3.4) holds on $|0\rangle_{t_{0}}$. The general proof of (3.4) is then obvious.

Equations (3.6) and (2.11) allow us to evaluate the average number of particles in the time-evolved vacuum, given by (3.3)

$$
\left\langle N_{t}\right\rangle=2 \sum_{i=1}^{\infty} \frac{\lambda_{i}^{2}}{1-\lambda_{i}^{2}}<\infty,
$$

which proves that the average number of particles is finite.

The average charge in $|0\rangle_{t t_{0}}$ is zero but the average charge-density is not. The expectation-value of the current (this has a well-defined meaning because the current is defined as a sesquilinear form, see (1.15)) is given by

$$
{ }_{t t_{0}}\left\langle 0\left|J_{t}^{\mu}(x)\right| 0\right\rangle_{t t_{0}}=\sum_{ \pm} \sum_{i=1}^{\infty} \frac{\lambda_{i}^{2}}{1-\lambda_{i}^{2}} j_{i}^{\mu^{ \pm}}(x, t),
$$

where $j_{i}^{\mu \pm}(x, t)$ is the classical current given by

$$
j_{i}^{\mu \pm}(x, t)=-i\left(\partial^{\mu} u_{i}^{ \pm}\right)^{*} u_{i}^{ \pm}+i u_{i}^{ \pm} * \partial^{\mu} u_{i}^{ \pm}-2 A^{\mu}\left|u_{i}^{ \pm}\right|^{2},
$$

with $f_{i}^{ \pm}=\left(\begin{array}{c}u_{i}^{ \pm} \\ i \partial_{t} u_{i}^{ \pm}\end{array}\right)$.

\section{III.4. Scattering Theory and Bound-State Problems}

In this section we shall consider vector-potentials $A_{\mu}(x, t)$ such that

$$
A_{\mu}(x, t)=A_{\mu}^{ \pm}(x) \text { for } t^{>}<t_{+}, t_{-} \leqq 0 \leqq t_{+} .
$$


Let us for $t \geqq t_{+}$introduce the following definitions

$$
\begin{gathered}
H_{ \pm}=H_{t}, \mathscr{H}_{ \pm}=\mathscr{H}_{t}, B_{ \pm}=B_{t}, W_{ \pm}=W_{ \pm}\left(B_{ \pm}, B_{0}\right) \\
\Psi_{ \pm}(f)=\Psi_{t}(f), \quad \Psi_{ \pm}^{\mathrm{as}}(f)=\Psi_{ \pm}\left(W_{ \pm} f\right),
\end{gathered}
$$

where $W_{ \pm}\left(B, B_{0}\right)$ is defined by (II.1.14).

We shall call $\Psi_{ \pm}^{\text {as }}(f)$ an asymptotic Schrödinger field, i.e.

$$
\Psi_{ \pm}^{\mathrm{as}}(f, t)=e^{i H_{ \pm} t} \Psi_{ \pm}^{\mathrm{as}}(f) e^{-i H_{ \pm} t}
$$

is a free field which is asymptotic in the following sense

$$
e^{i H_{ \pm} t} \Psi_{ \pm}(f) e^{-i H_{ \pm} t} \rightarrow \Psi_{ \pm}^{\mathrm{as}}(f, t), \quad t \rightarrow \pm \infty,
$$

strongly on any finite particle scattering subspace of $\mathscr{H}_{ \pm}$.

The $S$-operator is defined by

where

$$
\Psi_{+}(S f)=\mathscr{S} \Psi_{-}(f) \mathscr{S}^{-1},
$$

i.e.

$$
S=e^{-i B_{+} t+} \bar{U}\left(t_{+}, t_{-}\right) e^{i B_{-} t_{-}},
$$

$$
\mathscr{S}=e^{-i H_{+} t+} \mathscr{U}\left(t_{+}, t_{-}\right) e^{i H_{-} t_{-}},
$$

and is a unitary and charge-isometric operator from $\mathscr{H}_{-}$to $\mathscr{H}_{+}$.

Equation (4.4) can be written

$$
\begin{aligned}
& \Psi_{+}\left(S_{d d} f_{-}^{d}\right)+\Psi_{+}^{\mathrm{as}}\left(S_{c d} f_{-}^{d}\right)=\mathscr{S} \Psi_{-}\left(f_{-}^{d}\right) \mathscr{S}^{-1}, \\
& \Psi_{+}\left(S_{d c} f_{-}^{c}\right)+\Psi_{+}^{\mathrm{as}}\left(S_{c c} f_{-}^{c}\right)=\mathscr{S} \Psi_{-}^{\mathrm{as}}\left(f_{-}^{c}\right) \mathscr{S}^{-1},
\end{aligned}
$$

where $f_{-}^{c}=W_{-} f, f_{ \pm}^{d}=\left(E_{ \pm}(m)-E_{ \pm}(-m)\right) f=P_{ \pm}^{d} f\left(B_{ \pm}=\int \lambda d E_{ \pm}(\lambda)\right)$ and

$$
\begin{array}{ll}
S_{d d}=P_{+}^{d} S P_{-}^{d}, & S_{c d}=W_{+}^{*} S P_{-}^{d} \\
S_{d c}=P_{+}^{d} S W_{-}, & S_{c c}=W_{+}^{*} S W_{-} .
\end{array}
$$

The asymptotic initial (final) scattering states are defined by acting with monomials of $\Psi_{-}^{\text {as }}(f)\left(\Psi_{+}^{\text {as }}(f)\right)$ and $\Psi_{-}^{\text {as }}(f)^{*}\left(\Psi_{+}^{\text {as }}(f)^{*}\right)$ on the vacuum $|0\rangle_{-}\left(|0\rangle_{+}\right)$. The particle interpretation is obvious because $\Psi_{ \pm}^{\text {as }}(f)$ is a free Schrödinger field.

One can easily obtain $\mathscr{S}|0\rangle_{\text {_ }}$ (usually called the out-vacuum) by letting

$$
\bar{U}\left(t, t_{0}\right) \rightarrow e^{-i B_{+} t_{+}} \bar{U}\left(t_{+}, t_{-}\right) e^{i B_{-} t_{-}}
$$

in (III.2.9)-(III.2.13), i.e.

$$
\mathscr{S}|0\rangle_{-}=c \prod_{i=1}^{\infty} e^{-i \lambda_{\imath} a_{l}^{+*} a_{\imath}^{-*}}|0\rangle_{+},
$$


where $a_{i}^{ \pm}$can be expressed as follows

$$
\begin{gathered}
a_{i}^{ \pm}=a_{ \pm}^{\text {as }}\left(W_{ \pm}^{*}\left(B_{+}, B_{0}\right) f_{i}^{ \pm}\right)+a_{ \pm}\left(P_{+}^{d} f_{i}^{ \pm}\right), \\
a_{ \pm}^{\text {as }}(f)=a_{ \pm}\left(W_{ \pm}\left(B_{+}, B_{0}\right) f\right) .
\end{gathered}
$$

Any scattering amplitude (also involving eventual bound-states in the initial and/or final state) can be calculated by using Eqs. (4.7), (4.8), (4.10), and (4.11).

\section{III.5. Some Remarks on the D.H.H.P. and the D.H.S.P.}

We shall in this section give the connection between the diagonal Hamiltonian Heisenberg (Schrödinger) picture and the ordinary Heisenberg (Schrödinger) picture in a theory where both exists (they don't in our case).

Let $H_{S}(t)$ be a Hamiltonian (in the Schrödinger picture) defined in a Hilbert space $\mathscr{H}$. The time-evolution operator $U\left(t, t_{0}\right)$ in the Schrödinger picture (S.P.) is a solution of

$$
i \frac{\partial}{\partial t} U\left(t, t_{0}\right)=H_{S}(t) U\left(t, t_{0}\right),
$$

with the initial condition $U\left(t_{0}, t_{0}\right)=1$.

Let $A_{S}$ be a time-independent operator in the S.P. and define

$$
A_{H}(t)=U\left(t, t_{0}\right)^{-1} A_{S} U\left(t, t_{0}\right),
$$

to be the operator in the Heisenberg picture (H.P.) corresponding to $A_{S}$ in the S.P.

One can easily verify that $A_{H}(t)$ fulfills

$$
i \frac{d}{d t} A_{H}(t)=\left[A_{H}(t), H_{H}(t)\right],
$$

where

$$
H_{H}(t)=U\left(t, t_{0}\right)^{-1} H_{S}(t) U\left(t, t_{0}\right) .
$$

Let $V(t)$ be the unitary operator that diagonalizes $H_{H}(t)$, i.e. $V^{-1} H_{H} V$ is diagonal and put

$$
A_{D H}(t)=V(t)^{-1} A_{H}(t) V(t) .
$$

We then get in analogy with (6.3)

$$
i \frac{d}{d t} A_{D H}=\left[A_{D H}, H_{D H}\right]+i \frac{\partial}{\partial t} A_{D H},
$$


where

$$
H_{D H}=V^{-1} H_{H} V, \frac{\partial}{\partial t} A_{D H}=\left(\frac{\partial}{\partial t} V^{-1}\right) A_{H} V+V^{-1} A_{H} \frac{\partial}{\partial t} V .
$$

We then put

$$
\left(\frac{d}{d t}-\frac{\partial}{\partial t}\right) A_{D H}=\frac{d^{\prime}}{d t} A_{D H}
$$

and (6.6) can thus be written

$$
i \frac{d^{\prime}}{d t} A_{D H}=\left[A_{D H}, H_{D H}\right]
$$

Let us furthermore write

where

$$
A_{D H}(t)=\mathscr{U}\left(t, t_{0}\right) A_{D S}\left(t_{0}\right) \mathscr{U}\left(t, t_{0}\right),
$$

$$
\mathscr{U}\left(t, t_{0}\right)=V(t)^{-1} U\left(t, t_{0}\right)^{-1} V\left(t_{0}\right), A_{D S}\left(t_{0}\right)=V\left(t_{0}\right)^{-1} A_{S} V\left(t_{0}\right) .
$$

Equation (5.8) is the analog of (III.2.1) and (5.9) is the analog of (III.2.6).

Equation (5.10) gives the connection between the time-evolutionoperator (and an observable) in the D.H.S.P. and the S.P.

\section{Conclusions}

We have constructed a Hamiltonian formulation of relativistic quantum field theory for a charged spinless boson-field in interaction with a local external vector potential.

Guided by a diagonalized form of the classical field-energy we constructed a time-dependent canonical pair of Schrödinger fields $\Phi_{t}(x)$ and $\Pi_{t}(x)$ which diagonalized the field-Hamiltonian $H_{t}$.

These Schrödinger fields do in general belong to inequivalent representations of the canonical commutation relations for different $t$ 's.

The Heisenberg field was constructed by solving the Heisenberg equation of motion and its time-evolution turned out to be governed by a unitary operator i.e. the Heisenberg fields at different times are unitarily equivalent.

The time-evolution of an initial state was considered in a Schrödinger representation and quantum theory was developed to the same level of logical consistency as is possible in the corresponding non-relativistic problem.

The Dirac case (spin 1/2) can be treated in complete analogy (see Appendix C). 
Appendix A: Assumptions on the Local External Vector-potential $\boldsymbol{A}_{\mu}(\boldsymbol{x}, \boldsymbol{t})$

We shall give sufficient conditions on $A_{\mu}(x)$ such that an analysis like in [14] can be carried through (in [14] only the case $A_{\mu}(x)=\delta_{\mu 0} A_{0}(x)$ was considered). The following will be assumed;

i) $A_{\mu}(x)$ and $\boldsymbol{\nabla} \cdot \boldsymbol{A}(x)$ are real-valued and locally Hölder-continuous except at a finite number of singularities,

ii) $A_{0}^{2}(x)$ is square integrable and $A(x)$ is bounded,

iii) $A_{\mu}(x)$ and $\boldsymbol{\nabla} \cdot \boldsymbol{A}(x)$ behave as $\mathcal{O}\left(|x|^{-3-\varepsilon}\right), \varepsilon>0,|x| \rightarrow \infty$,

iv) $\int d x\left(A^{2}|f|^{2}+\boldsymbol{A}\left(i(\boldsymbol{\nabla} f)^{*} f-f^{*} i \nabla f\right)\right)$

$$
\leqq \alpha \int d x\left(|\nabla f|^{2}+m^{2}|f|^{2}\right), 0<\alpha<1, f \in C_{0}^{\infty}\left(R^{3}\right) .
$$

All parts of [14] goes straight through in this more general case except Remark 4.3 where some work has to be done. More precisely in the analysis of the Lippmann-Schwinger equation (II.1.9) one has to use a Banach-space $B$ whose norm contains the gradient operator, i.e.

$$
\|u\|_{B}=\sup _{x}(|u(x)|+|\nabla u(x)|) .
$$

Once this Banach-space is employed the analysis becomes completely analogous to the case $\boldsymbol{A}=0$.

The time-dependent potential $A_{\mu}(x, t)$ is assumed to fulfill the conditions above uniformly in $t$ and furthermore we assume that $A_{\mu}(x, t)$ is continuous in $t$ uniformly in $x$ and that $\partial_{t} A_{\mu}(x, t)$ is integrable over $R^{3}$ and locally integrable over $R^{1}$.

\section{Appendix B. Unitary of $U\left(t, t_{0}\right)$}

In the end of Section III.3 we argued that it was sufficient for the unitarity of $\mathscr{U}\left(t, t_{0}\right)$ to hold that

i) $\bar{U} \mathscr{H}_{I_{t_{0}}}^{+}$is dense in $\mathscr{H}_{I_{t}}^{+}$,

ii) $\|K\|_{I_{t}}<1$,

iii) $K$ is Hilbert-Schmidt in $\mathscr{H}_{I_{t}}$.

Proof of i). Let's assume the contrary, i.e. there exists a $g^{+} \in \mathscr{H}_{I_{t}}^{+}$ such that (we put $I_{t}=I$ and $I_{t_{0}}=I_{0}$ )

$$
\left(g^{+}, \bar{U} f_{0}^{+}\right)_{I}=0, \text { for all } f_{0}^{+} \in \mathscr{H}_{I_{0}}^{+}
$$

and thus $\left(\bar{U}^{-1} g^{+}, f_{0}^{+}\right)_{I_{0}}=0$ due to the charge-isometry of $\bar{U}$. This means that

and thus

$$
h_{0}^{-}=\bar{U}^{-1} g^{+} \in \mathscr{H}_{I_{0}}^{-}
$$

$$
\left(\bar{U}^{-1} g^{+}, \bar{U}^{-1} g^{+}\right)_{Q_{0}}=-\left\|h_{0}^{-}\right\|_{I_{0}}^{2}=\left\|g^{+}\right\|_{I}^{2},
$$

which proves that $g^{+}=0$. Q.E.D. 
Proof of ii). We have

$$
\bar{U} f_{0}^{+}=g^{+}+K g^{+},
$$

with $g^{+}=P^{+} \bar{U} f_{0}^{+}$and thus the following identity holds

$$
\left(\bar{U} f_{0}^{+}, \bar{U} f_{0}^{+}\right)_{Q}=\left(f_{0}^{+}, f_{0}^{+}\right)_{Q_{0}}=\left(g^{+}, g^{+}\right)_{Q}+\left(K g^{+}, K g^{+}\right)_{Q},
$$

and therefore

$$
\left\|K g^{+}\right\|_{I}^{2}=\left\|g^{+}\right\|_{I}^{2}-\left\|f_{0}^{+}\right\|_{I_{0}}^{2}>0
$$

i.e. $\|K\|_{I}<1$ due to i).

Proof of iii). We shall prove the Hilbert-Schmidt property of $K$ given by

$$
\begin{gathered}
K=\left(1-K_{1}\right)^{-1} K_{2}, \\
K_{1}=P^{-} \bar{U} P_{0}^{+} \bar{U}^{-1}, \quad K_{2}=P^{-} \bar{U} P_{0}^{+} \bar{U}^{-1} P^{+},
\end{gathered}
$$

by establishing the Hilbert-Schmidt property of $\bar{K}=P^{-} \bar{U} P_{0}^{+}$as an operator from $\mathscr{H}_{I_{0}}$ to $\mathscr{H}_{I}$ in perturbation theory $\left(\left(1-K_{1}\right)^{-1}\right.$ is bounded because $K_{1}$ is negative and $\bar{U}^{-1} P^{+}$is also obviously bounded). $\bar{U}\left(t, t_{0}\right)$

In analogy with (II.2.25) we have the following representation for

with

$$
\widetilde{U}\left(t, t_{0}\right)=e^{i B_{t_{0}}\left(t-t_{0}\right)} T e^{i \int_{t_{0}}^{t} d t \bar{V}_{t t_{0}}},
$$

$$
\bar{V}_{t t_{0}}=e^{-i B_{t_{0}}\left(t-t_{0}\right)} V_{t t_{0}} e^{i B_{t_{0}}\left(t-t_{0}\right)},
$$

where $V_{t t_{0}}=\bar{B}_{t}-B_{t_{0}}\left(\right.$ for $\bar{B}_{t}$ see (III.2.5)) is bounded in $\mathscr{H}_{I_{t_{0}}}$ (follows from the assumptions on $A_{\mu}$ given in II.2).

The Hilbert-Schmidt (H.S.) property of $K$ will be proved by making a perturbation expansion of (B.3)

$$
\bar{U}\left(t, t_{0}\right)=e^{i B_{t_{0}}\left(t-t_{0}\right)}\left(1+i \int_{t_{0}}^{t} d t \bar{V}_{t t_{0}}+\cdots\right) .
$$

By using the explicit representation (I.1.10) of the spectral projections one can verify that it is necessary and sufficient for $\bar{K}\left(t, t_{0}\right)$ to be a H.S. operator from $\mathscr{H}_{I_{t_{0}}}$ to $\mathscr{H}_{I_{t}}$ that

$$
F\left(k, k^{\prime}\right)=\left(\Phi_{t}^{-}(\cdot, k), \bar{U}\left(t, t_{0}\right) \Phi_{t_{0}}^{+}\left(\cdot, k^{\prime}\right)\right)_{\mathscr{E}_{t}},
$$

fulfills

$$
\int d k d k^{\prime} \frac{\omega_{k}}{\omega_{k^{\prime}}}\left|F\left(k, k^{\prime}\right)\right|^{2}<\infty .
$$


By inserting (B.5) into (B.6) we get to lowest order in $V_{t t_{0}}$

$$
\begin{aligned}
F\left(k, k^{\prime}\right)= & \left(\Phi_{t}^{-}(\cdot, k), \Phi_{t_{0}}^{+}\left(\cdot, k^{\prime}\right)\right)_{\mathscr{E}_{t}} e^{i \omega_{k^{\prime}}\left(t-t_{0}\right)} \\
& +i\left(\Phi_{t}^{-}(\cdot, k), \int_{t_{0}}^{t} d t \bar{V}_{t t_{0}} \Phi_{t_{0}}^{+}\left(\cdot, k^{\prime}\right)\right)_{\mathscr{E}_{t}} e^{-i \omega_{k}\left(t-t_{0}\right)} \\
= & F_{1}\left(k, k^{\prime}\right)+F_{2}\left(k, k^{\prime}\right) .
\end{aligned}
$$

The following equation holds when interpreted properly

where

$$
\Phi_{t_{0}}^{+}(\cdot, k)=\Phi_{t}^{+}(\cdot, k)+R_{t}\left(\omega_{k}+i 0\right) V_{t t_{0}}^{\prime} \Phi_{t_{0}}^{+}(\cdot, k),
$$

$$
V_{t t_{0}}^{\prime}=B_{t}-B_{t_{0}}, \quad R(z)=\frac{1}{B_{t}-z} .
$$

The first term in (B.8) can now be rewritten by using (B.9)

$$
F_{1}\left(k, k^{\prime}\right)=-\frac{1}{\omega_{k}+\omega_{k^{\prime}}}\left(\Phi_{t}^{-}(\cdot, k), V_{t t_{0}}^{\prime} \Phi_{t_{0}}^{+}\left(\cdot, k^{\prime}\right)\right)_{\mathscr{E}_{t}} e^{i \omega_{k^{\prime}}\left(t-t_{0}\right)} .
$$

The second term in (B.8) can be written as follows

$$
F_{2}\left(k, k^{\prime}\right)=i\left(\Phi_{t}^{-}(\cdot, k), \int_{t_{0}}^{t} d t e^{i\left(\omega_{k}+\omega_{k^{\prime}}\right)\left(t-t_{0}\right)} V_{t t_{0}} \Phi_{t_{0}}^{+}\left(\cdot, k^{\prime}\right)\right){ }_{\mathscr{E}_{t}} e^{-i \omega_{k_{k}}\left(t-t_{0}\right)},
$$

plus terms of higher order in $V$.

We now use the fact that $\Phi_{t}^{+}(x, k)$ has the form (II.1.7) where $u_{t}^{+}(x, k)$ has the property

$$
u_{t}^{+}(x, k) \rightarrow e^{i k x} \text { when } k \rightarrow \infty,
$$

for our choice of potentials.

It is now straight forward to verify that $F=F_{1}+F_{2}$ fulfills (B.7) to first order in $V_{t t_{0}}$ by employing partial integration in (B.12) and using the continuity of $V_{t t_{0}}$ as a function of $t$. The verification of (B.7) in higher orders is straight-forward and the perturbation expansion is known to be convergent which finally proves the Hilbert-Schmidt property of $K=K\left(t, t_{0}\right)$.

\section{Appendix C. The Dirac Case}

The Dirac case is actually simpler than the Klein-Gordon case once the classical problem is solved (spectral and scattering theory).

The reason for this is that the Dirac equation can be written

$$
i \partial_{t} \psi=H_{\mathrm{cl}} \psi \text {, }
$$


where

$$
H_{\mathrm{cl}}=-i \boldsymbol{\alpha} \cdot \boldsymbol{D}+A_{0}+\beta m, \quad \boldsymbol{D}=\boldsymbol{\nabla}+i \boldsymbol{A},
$$

and is self-adjoint on $\mathscr{H}_{\mathrm{cl}}=L^{2} \oplus L^{2} \oplus L^{2} \oplus L^{2}$, i.e. the classical Hilbertspace is time-independent and the time-evolution operator is unitary in $\mathscr{H}_{\mathrm{cl}}$.

The quantization can then be carried through in complete analogy with the K-G case.

Acknowledgements. I want to thank Professors J. S. Bell, L. Gårding, R. Haag and J. Hamilton for stimulating discussions. Also, I am grateful for the hospitality shown to me at Nordita.

\section{References}

1. Heisenberg, W., Pauli, W.: Zeitschrift für Physik 56, 1 (1929).

2. Schnyder, H., Weinberg, J.: Phys. Rev. 57, 307 (1940).

3. Moses, H.E.: Phys. Rev. 89, 115 (1953), 95, 237 (1954).

4. Bongaarts, P. J. M.: Ann. of Phys. 56, 108 (1970).

5. Lehmann, H., Symanzik, K., Zimmermann, W.: Nuovo Cimento 1, 425 (1955).

6. Feynmann, R. P.: Phys. Rev. 76, 749 (1949).

7. Dyson, F. J.: Phys. Rev. 75, 486 (1949).

8. Salam, A., Matthews, P.T.: Phys. Rev. 90, 690 (1953).

9. Schwinger, J.: Phys. Rev. 93, 615 (1954).

10. Capri, A.Z.: Journ. Math. Phys. 10, 575 (1969).

11. Schroer, B., Seiler, R., Swieca, J. A.: Phys. Rev. D, 2, 2929 (1970).

12. Ekstein, H.: Phys. Rev. D, 5, 5 (1972).

13. Labonté, G., Capri, A. Z.: Nuovo Cimento B 10, 583 (1972).

14. Lundberg, L.-E.: Commun. math. Phys. 31, 243-257 (1973).

L.-E. Lundberg

Nordita

Blegdamsvej 17

Kopenhagen, Denmark 